\title{
Efeito da Pastagem, da Produção e da Composição do Leite no Desempenho de Bezerros de Diferentes Grupos Genéticos
}

\author{
João Restle ${ }^{1}$, Paulo Santana Pacheco ${ }^{2}$, Leonir Luiz Pascoal ${ }^{3}$, João Teodoro Pádua ${ }^{4}$, José \\ Luiz Moletta ${ }^{5}$, Aline Kellermann de Freitas ${ }^{6}$, Daniel Terra Leite $^{7}$
}

\begin{abstract}
RESUMO - O experimento foi conduzido com o objetivo de avaliar o desempenho de bezerros de corte dos grupos genéticos Charolês

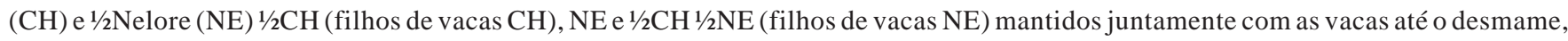
em pastagem nativa (PN) ou pastagem cultivada (PC) de inverno (aveia + azevém) e de verão (capim-papuã). Foram utilizados sessenta bezerros entre puros e mestiços, pesados ao nascimento, 14, 42, 70, 98, 126, 154, 182 dias e ao desmame. A produção de leite das vacas foi avaliada na mesma data das pesagens, com exceção do nascimento. Bezerros mantidos em PC apresentaram maior ganho de peso médio diário (GMD) (733 contra 440 g) e maior peso ao desmame (191,8 contra 128,6 kg) que os mantidos em PN. Foi verificado efeito quadrático do período pós-parto no GMD ( $y=489,89$ + 3,96DIA - 0,0198DIA²), sendo que o ponto máximo do GMD (571 g) foi aos 85 dias de idade para os mantidos em PN e aos 107 dias (848 g) para os mantidos em PC. A produção média diária de leite das vacas explicou 45\% do peso ao desmame e 38\% do GMD dos bezerros. As correlações entre a produção de leite e o GMD e o peso dos bezerros foi significativa mesmo nos estágios mais avançados de lactação. A correlação entre a produção média diária de leite de todo período de aleitamento e o GMD foi maior nos animais mantidos em PC $(0,54)$ do que em PN $(0,44)$ e nos animais F1 $(0,73)$ em relação aos puros $(0,47)$. A menor correlação entre produção de leite e GMD foi verificada nos bezerros NE puros (0,31), nos demais foi de 0,60; 0,71; e 0,84, para CH, $1 / 2 \mathrm{CH} 1 / 2 \mathrm{NE}$ e $1 / 2 \mathrm{NE} 1 / 2 \mathrm{CH}$. Os teores de gordura, lactose, extrato seco desengordurado e extrato seco total, não apresentaram correlação significativa com o GMD. No entanto, quando foi considerada a produção total desses constituintes do leite, as correlações com o GMD foram significativas. Bezerros F1 apresentaram maiores pesos ao nascimento e ao desmame que os puros. A heterose para o peso ao nascimento, desmame e GMD foi, respectivamente, de 3,4; 11,8; e 10,2\%.
\end{abstract}

Palavras-chave: Charolês, cruzamento, ganho de peso, heterose, idade da vaca, Nelore

\section{Effect of Pasture, Milk Yield and Composition on the Performance of Beef Calves from Different Genetic Groups}

\begin{abstract}
The objective of the experiment was to evaluate the performance of Charolais $\left(\mathrm{CH}\right.$ ) and $1 / 2 \mathrm{Nellore}$ (NE) $\frac{1}{2} \mathrm{CH}$ (produced by $\mathrm{CH}$ cows), $\mathrm{NE}$ and $1 / 2 \mathrm{CH} 1 / 2 \mathrm{NE}$ (produced by $\mathrm{NE}$ cows) calves, mantained with the cows until weaning, on native pasture (NP) or winter (oat + italian rye grass) and summer (alexander grass) cultivated pasture (CP). Sixty straigthbred or crossbreds calves were used, and weighted at birth; 14; 42; 70; 98; 126; 154; 182 days; and at weaning. The cows milk yield was evaluated at the same date of the calves weighing, with exception at birth. Calves mantained on CP showed higher average daily weight gain (ADG) (733 vs 440 g) and were heavier at weaning (191,8 vs 128,6 kg) than calves mantained on NP. Quadratic effect was observed for calves ADG (y=489.89 + 3.96DAY - 0.0198DAY2), being the ADG maximum point (571 g) at 85 days of age for those mantained on NP and at 107 days (848 g) for those mantained on CP. Average daily milk yield explained $45 \%$ of calves weaning weight and $38 \%$ of the ADG. The correlations between the milk yield and calves ADG and weight were significant even in the advanced stages of lactation. The correlation between the average daily milk yield during the whole lactation period and the ADG was higher in animals mantained on CP (.54) than on NP (.44) and for F1 animals (.73) in relation to straigthbreds (.47). The lowest correlation between milk yield and ADG was observed for straigthbred NE calves (.31), for the other groups the correlation were .60; .71; and .84, for $\mathrm{CH} ; 1 / 2 \mathrm{CH} 1 / 2 \mathrm{NE}$ and $1 / 2 \mathrm{NE} 1 / 2 \mathrm{CH}$, respectively. Fat, lactose, nonfat dry extract and total dry extract contents did not show significant correlation with the ADG. However, when the total production of these components were considered, the correlations with ADG were significant. F1 calves were heavier at birth and weaning than straigthbreds. The heterosis for birth weight, weaning weight and ADG was, respectively, 3.4; 11.8; and 10.2\%.
\end{abstract}

Key Words: Charolais, crossbred, weight gain, heterosis, cow age, Nellore

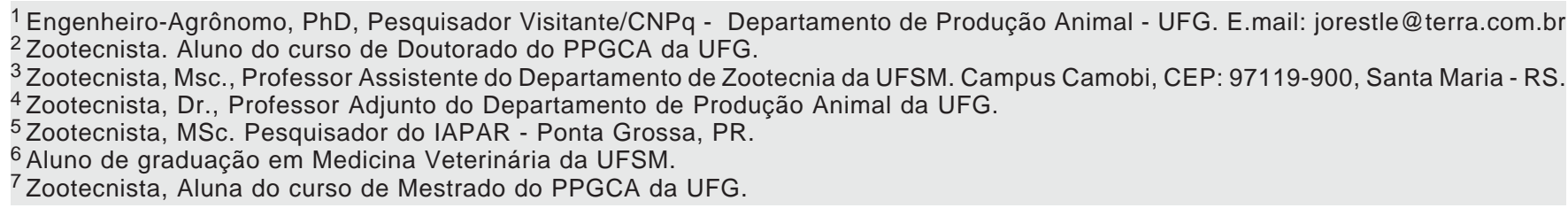




\section{Introdução}

As propriedades voltadas para a exploração da bovinocultura de corte enfrentam cada vez mais dificuldades para se manter competitivas em um mercado que está sempre a exigir maiores ganhos de eficiência, redução de custos e aumento de produtividade. Em sistema de ciclo completo, uma alternativa para maximizar a rentabilidade seria aumentar a quantidade de kg de bezerro desmamado por vaca/ ano, que é determinada pela taxa de desmame e pelo peso médio dos bezerros.

Peso ao desmame é uma característica importante, pois reflete o potencial genético do terneiro e a habilidade materna da vaca. À medida que a produção de gado de corte é intensificada, o peso ao desmame se torna cada vez mais importante, pois está associado com a idade à puberdade das fêmeas e a idade de abate dos machos (Restle et al., 1999a; Restle et al., 1999b). Segundo Ribeiro \& Restle (1991), existem vários fatores considerados importantes que atuam sobre o ganho de peso pré-desmame e sobre o peso ao desmame do bezerro, destacando-se a habilidade materna, que é determinada principalmente pela produção leiteira da vaca, o potencial genético para ganho de peso, o sexo e o nível nutricional.

Quanto ao efeito da produção de leite da vaca no desenvolvimento do bezerro, normalmente vacas de corte com maior produção de leite produzem bezerros que apresentam melhores ganhos de peso até o desmame. Segundo diversos autores, a correlação entre a produção de leite da vaca e o peso ao desmame do bezerro é positiva e alta, acima de 0,50 (Melton et al., 1967; Totusek et al., 1973; Ribeiro \& Restle, 1991).

Já os fatores que influenciam o potencial para ganho de peso dos bezerros são o grupo genético, a heterozigose e a seleção dentro do rebanho. Dados de diversas pesquisas têm comprovado as maiores taxas de ganho de peso e pesos à desmama em animais mestiços (Crockett et al., 1978; Reynolds et al., 1978; Rocha \& Ribeiro, 1987; Becker et al., 1995).

Quanto ao sexo do bezerro, trabalhos de pesquisa mostram maiores ganhos de peso e maiores pesos ao desmame para os machos em relação às fêmeas (Melton et al., 1967; Bond \& Wiltbank, 1970; Rutledge et al., 1971; Cundiff et al., 1974; Reynolds et al., 1978; Ribeiro \& Restle, 1991). Porém, Bellows \& Short (1978) e Rocha \& Ribeiro (1987) não encontraram diferença entre os sexos no peso à desmama.

De acordo com Neville Jr. (1962), Bond \&
Wiltbank (1970), Rovira (1974) e Costa et al. (1981), a nutrição é um fator importante para o desempenho tanto da vaca quanto do bezerro. Vacas em melhores condições alimentares produzem mais leite e, conseqüentemente, bezerros mais pesados ao desmame, além de que com as mudanças anatômicas, fisiológicas e metabólicas que ocorrem no sistema digestivo do ruminante jovem, ao redor dos 4 meses de idade, o ganho de peso dos bezerros depende mais da ingestão de forragem que da ingestão de leite, reduzindo a correlação entre produção de leite e ganho de peso médio diário a partir desta idade (Robison et al., 1978; Leal \& Freitas, 1982).

O estudo de Moojen et al. (1994 a,b), em condições sul brasileiras, comprovou que quando as condições alimentares foram melhoradas no período pós-parto (pastagem cultivada de inverno), tanto vacas como bezerros apresentaram melhores desempenhos em relação àqueles animais mantidos em pastagem nativa.

O presente estudo foi conduzido com o objetivo de avaliar o desempenho de bezerros puros e F1 1/2 Nelore $1 / 2$ Charolês filhos de vacas Charolês, e de puros e F1 $1 / 2$ Charolês $1 / 2$ Nelore, filhos de vacas Nelore, submetidos a dois tipos de pastagem, pastagem nativa ou cultivada de inverno e verão, do nascimento ao desmame. Estudou-se também a relação entre a produção e constituintes do leite com o desempenho dos bezerros.

\section{Material e Métodos}

O experimento foi conduzido no Setor de Bovinocultura de Corte do Departamento de Zootecnia da Universidade Federal de Santa Maria, no município de Santa Maria, Rio Grande do Sul, na região fisiográfica Depressão Central, que, segundo classificação de Köppen, apresenta clima subtropical úmido (cfa).

Foram utilizados 60 pares de vacas e bezerros contemporâneos, tomados ao acaso do mesmo rebanho experimental, sendo 15 do grupo genético Charolês (CH) e 15 do grupo genético $1 \frac{1}{2} \mathrm{NE} 1 \frac{1}{2} \mathrm{CH}$ (filhos de vacas $\mathrm{CH}$ ), 15 do grupo genético Nelore (NE) e 15 do grupo genético $1 \frac{1}{2} \mathrm{CH} 1 / 2 \mathrm{NE}$ (filhos de vacas $\mathrm{NE}$ ), submetidos a uma das seguintes pastagens: pastagem nativa (PN) do nascimento ao desmame ou pastagem cultivada (PC) de aveia (Avena sativa) + azevém (Lollium multiflorum) do nascimento até o final do mês de novembro e após em pastagem de capimpapuã (Brachiaria plantagínea), até o desmame.

O período de parição foi de setembro a dezembro, sendo os bezerros obtidos de inseminação artificial 
(período de 45 dias) e de monta natural (período de 45 dias). Durante o período de inseminação, foi utilizado o sêmen de oito touros $\mathrm{CH}$ e NE e, para o repasse, quatro touros $\mathrm{CH}$ e quatro NE. Os touros que produziram os bezerros $\mathrm{CH}$ foram os mesmos que produziram os $1 / 2 \mathrm{CH}$ $1 / 2 \mathrm{NE}$ e os que produziram os bezerros NE, os mesmos que produziram os $1 / 2 \mathrm{NE} 1 / 2 \mathrm{CH}$.

Nos tratamentos, os animais ficaram equilibrados pela ordem de parição e idade da vaca.

Foi utilizada uma área de 17 ha, com pastagem de inverno (aveia + azevém) e outros 17 ha com pastagem de verão (capim-papuã), sendo a lotação/ha de 1,76 vacas com bezerro ao pé, em ambas PC de inverno e verão. Para os animais mantidos em $\mathrm{PN}$, foi utilizada uma área de 30 ha, sendo a lotação/ha de 1,0 vaca com bezerro ao pé.

O critério adotado para estabelecer as lotações em pastagem nativa foi por esta representar a lotação média utilizada na região sul do país neste período. $\mathrm{Na}$ pastagem cultivada, baseou-se em resultados de pesquisas realizadas em condições sul-brasileiras, envolvendo as mesmas espécies forrageiras.

Avaliou-se a massa de forragem disponível por intermédio da técnica da dupla amostragem (Wilm et al., 1944). Foram coletadas amostras da forragem a cada quatro semanas aproximadamente, cortadas rente ao solo, em uma área de $0,25 \mathrm{~m}^{2}$, para posterior determinação da matéria seca (MS), em estufa de ar forçado a $55^{\circ} \mathrm{C}$ por 72 horas, digestibilidade in vitro da matéria orgânica (DIVMO), conforme Tilley \& Terry (1963), e da proteína bruta (PB), pelo método micro kjeldahl, segundo AOAC (1984).

As espécies forrageiras predominantes na pastagem nativa verificadas foram Andropogon lateralis Nees., Paspalum notatum Fl. e Eragrostis plana Nees. (capim-Annoni 2).

Os bezerros foram pesados nas primeiras $24 \mathrm{~h}$ pós-parto; aos 14; 42; 70; 98; 126; 154; 182 dias; e ao desmame, que ocorreu com média de 217 dias de idade.

Foi calculada a heterose $(\mathrm{H})$ para as características peso ao nascer, peso ao desmame, e ganho de peso médio diário do nascimento ao desmame, por meio da seguinte fórmula: $\mathrm{H}(\%)$ = (média dos F1/ média dos puros -1$) * 100$.

Também foram verificadas as produções de leite das vacas, utilizando o método direto, com ordenha manual, seguindo a técnica descrita por Ribeiro et al. (1991). Posteriormente à ordenha, foram coletadas amostras de $300 \mathrm{~mL}$, para determinação, em laboratório dos componentes do leite: percentagem de gor- dura, lactose, extrato seco total e extrato seco desengordurado. As avaliações foram realizadas aos 14 dias pós-parto e a partir daí com intervalos de 28 dias até o desmame dos bezerros. Os resultados da produção e composição do leite foram relatados em Restle et al. (2003) e são utilizados neste trabalho para análises de correlação.

O delineamento experimental utilizado foi o inteiramente casualizado, sendo os dados submetidos à análise de variância, teste $\mathrm{F}$ e teste Tukey quando o F foi significativo a 5\%, por intermédio do programa estatístico SAS (1997). O modelo estatístico utilizado foi:

$$
\begin{gathered}
\Upsilon_{i j k l m n o p}=\mu+\mathrm{NT}_{i}+\mathrm{GG}_{j}+\mathrm{P}_{k}+\mathrm{SA}_{p}+\mathrm{RT}_{l}(\mathrm{SA})_{\mathrm{p}}+ \\
\left(\mathrm{P}^{*} \mathrm{GG}\right)_{k j}+\mathrm{S}_{m}+\left(\mathrm{GG}^{*} \mathrm{~S}_{j m}+\mathrm{IV}_{n}+\left(\mathrm{IV} \mathrm{IV}_{n m}+\right.\right. \\
(\mathrm{IV} * \mathrm{P})_{n k}+(\mathrm{GG} \text { (GV) })_{j n}+\mathrm{DIA}_{o}+\left(\mathrm{P}^{*} \mathrm{DIA}\right)_{k o}+ \\
(\mathrm{GG} \text { DIA })_{j o}+\left(\mathrm{P}^{*} \mathrm{GG} \mathrm{GIA}_{k j o}+\mathrm{e}_{i j k l m n o p}\right.
\end{gathered}
$$

em que: $\Upsilon_{i j k l m n o}=$ variáveis dependentes; $\mu$ = média geral de todas as observações; $\mathrm{NT}_{i}=$ efeito da covariável "ordem de nascimento do bezerro" de ordem "i”; $\mathrm{GG}_{j}=$ efeito do grupo genético da vaca de ordem " $\mathrm{j}$ ", sendo $1=$ Charolês e $2=$ Nelore; $\mathrm{P}_{k}=$ efeito do tipo de pastagem de ordem " $k$ ", sendo $1=$ pastagem cultivada e 2=pastagem nativa; $\mathrm{SA}_{p}=$ efeito do sistema de acasalamento que originou os bezerros de ordem "p", sendo 1=puros e $2=$ mestiços; $\mathrm{RT}_{l}(\mathrm{SA})_{p}=$ efeito da raça do bezerro de ordem "l” sendo 1=Charolês, $2=$ =Nelore, 3=1/2Charolês $1 / 2$ Nelore e 4=1/2Nelore1/2Charolês dentro do sistema de acasalamento de ordem "p"; $\left(\mathrm{P}^{*} \mathrm{GG}\right)_{k j}=$ interação entre k-ésimo tipo de pastagem e j-ésimo grupo genético da vaca; $\mathrm{S}_{m}=$ efeito do sexo do bezerro de ordem "m", sendo 1=macho e $2=$ fêmea; $\left(G^{*} S\right)_{j m}=$ interação entre j-ésimo grupo genético da vaca e mésimo sexo do bezerro; $\mathrm{IV}_{n}=$ efeito da classe de idade da vaca de ordem “n”, sendo $1=$ jovens, $2=$ adultas e 3=velhas; $\left(\mathrm{IV}^{*} \mathrm{~S}\right)_{n m}=$ interação entre n-ésima idade da vaca e m-ésimo sexo do bezerro; $\left(\mathrm{IV}^{*} \mathrm{P}\right)_{n k}=$ interação entre n-ésima idade da vaca e k-ésimo tipo de pastagem; $\left(G^{*} I V\right)_{j n}=$ interação entre j-ésimo grupo genético da vaca e n-ésima idade da vaca; DIA $_{o}=$ efeito do período de lactação de ordem “o”; $(\mathrm{P} * \mathrm{DIA})_{k o}=$ interação entre o k-ésimo tipo de pastagem e o-ésimo período de lactação; $(\mathrm{GG} * \mathrm{DIA})_{j o}=$ interação entre o j-ésimo grupo genético da vaca e oésimo período de lactação; ( $\mathrm{P}^{*} \mathrm{GG}$ *DIA $)_{k j o}=$ interação entre k-ésimo tipo de pastagem, j-ésimo grupo genético da vaca e o-ésimo período de lactação; $\mathrm{e}_{i j k l m n o}=$ erro aleatório residual, NID $\left(0, \mathrm{~s}^{2}\right)$. 
Para estimativas das equações de regressão, o efeito de período (dias pós-parto) sobre as variáveis dependentes foi submetido à análise de regressão polinomial. Para a variável dependente peso do bezerro ao nascer, o efeito do tipo de pastagem foi retirado do modelo.

\section{Resultados e Discussão}

A massa média de forragem disponível na pastagem cultivada de inverno foi de $1.252 \mathrm{~kg}$ de MS/ha e na de verão, de 1.879,6 kg de MS/ha. Já na pastagem nativa foi de $1.654,4 \mathrm{~kg}$ de MS/ha. A digestibilidade in vitro da matéria orgânica (DIVMO) e o teor de proteína bruta (PB), citados na mesma ordem, foram de 59,4 e $9,7 \% ; 58,9$ e $6,1 \%$; 36,8 e $5,6 \%$. Verificou-se que animais mantidos em pastagem cultivada tiveram à disposição alimento de melhor qualidade, representado pela maior concentração de matéria orgânica digestível e de proteína bruta.

Apesar de não ter sido verificada interação significativa entre tipo de pastagem, grupo genético da vaca e período pós-parto para peso dos bezerros, na Figura 1 está representado o comportamento da evolução desta característica. Nota-se maior desenvolvimento dos bezerros filhos de vacas Charolês $(\mathrm{y}=32,74( \pm 0,25)+0,811( \pm 0,002)$ DIA; $C V=2,22 \%$; $\left.\mathrm{R}^{2}=0,99\right)$ e Nelore $(\mathrm{y}=22,18( \pm 0,26)+0,740( \pm$ 0,002)DIA; $\left.\mathrm{CV}=2,70 \% ; \mathrm{R}^{2}=0,99\right)$, quando mantidos em pastagem cultivada em relação aos bezerros filhos de vacas Charolês $(y=36,01( \pm 0,30)+0,515$ ( \pm 0,002)DIA; $\left.C V=3,53 \% ; R^{2}=0,99\right)$ e Nelore $(y=29,76$ $( \pm 0,42)+0,494( \pm 0,003)$ DIA; $\left.C V=5,44 \% ; \mathrm{R}^{2}=0,98\right)$ mantidos em pastagem nativa.

Na Tabela 1, são apresentados os pesos médios dos bezerros nos diferentes períodos avaliados. Houve interação significativa $(\mathrm{P}=0,0001)$ entre tipo de pastagem e período, sendo que a evolução do peso dos bezerros ao longo dos períodos foi linear para ambos tratamentos. Como pode ser verificado nas equações de regressão, bezerros mantidos em PC apresentaram GMD bem superior ao dos bezerros mantidos em PN.

Esse resultado demonstra que o uso de pastagem cultivada no período pós-parto promoveu maiores pesos à desmama dos bezerros, devido principalmente à maior produção de leite das vacas mantidas em PC $(4,80 \mathrm{~L} /$ dia $)$ em relação às mantidas em $\mathrm{PN}$ (3,98 L/dia), representando uma diferença de $21 \%$ (Restle et al., 2003). Esta constatação está de acordo com os comentários de Rutledge et al. (1971), Totusek et al. (1973) e Barcellos \& Lobato (1992) de que vacas de corte com maiores produções de leite produzem bezerros que apresentam melhores ganhos de peso. Além disso, a partir do momento em que os bezerros iniciaram a substituição do leite materno por forragem, tiveram à disposição alimento de melhor qualidade, representado pela maior concentração de MO digestível e de PB da pastagem cultivada, que foi de 59,4 e 9,7\%; 58,9 e 6,1\%, respectivamente, para PC de inverno e PC de verão, e 36,8 e 5,6\% para a PN. Segundo Costa et al. (1981) e Salomoni et al. (1982), em geral, as condições da pastagem nativa em certos períodos do ano são insuficientes em disponibilidade e qualidade para atender às necessidades nutricionais do rebanho de cria.

O estudo de Quadros \& Lobato (1997), que avaliou o efeito da lotação animal na produção de leite de vacas e no desenvolvimento de bezerros de corte, demonstrou que, quando os animais foram submetidos à menor lotação ( 0,6 equivalente vaca/ha), os bezerros apresentaram maiores pesos ao desmame em relação àqueles submetidos à lotação de 0,8 equivalente vaca/ha (161,0 vs 148,0 kg), resultado associado às diferenças na disponibilidade e qualidade ocorrida na pastagem nativa.

Na Tabela 2, encontram-se os valores referentes ao comportamento do ganho de peso médio diário dos bezerros de acordo com o tipo de pastagem, grupo genético da vaca e período pós-parto.

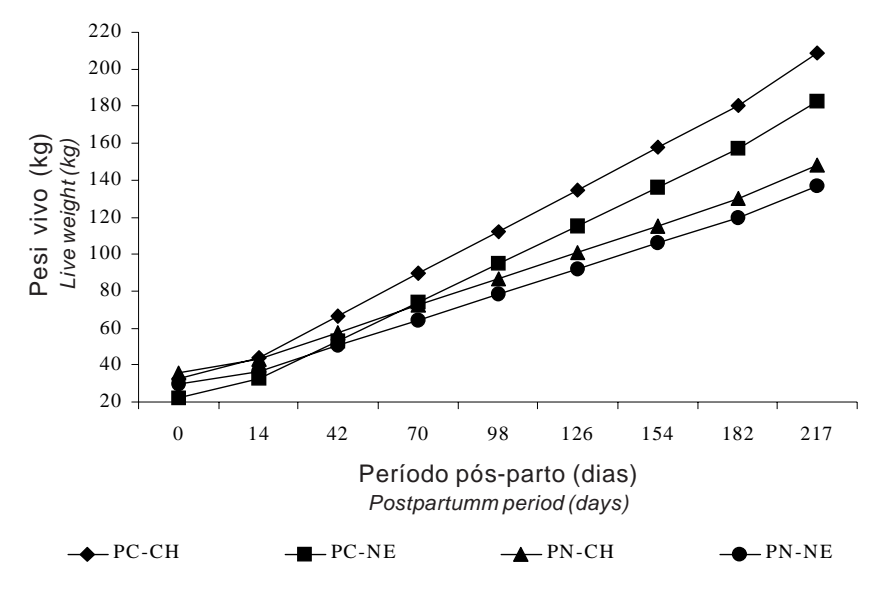

Figura 1 - Evolução do peso vivo dos bezerros de acordo com o grupo genético da vaca, tipo de pastagem e período pós-parto.

Figure 1 - Calf live weight evolution according to cow genetic group, pasture and postpartum period. 
Verificou-se que os bezerros mantidos em PN atingiram o ponto de GMD máximo (571 g) aos 85 dias de idade $(\mathrm{y}=397,21( \pm 6,58)+4,09( \pm 0,13)$ DIA - 0,024 ( \pm 0,001)DIA $\left.{ }^{2} ; \mathrm{CV}=10,5 \% ; \mathrm{R}^{2}=0,88\right)$. Já para os bezerros mantidos em PC o ponto de GMD máximo (848 g) foi atingido aos 107 dias da idade $(\mathrm{y}=518,46( \pm 13,1)+6,18( \pm 0,26)$ DIA $-0,029$ $( \pm 0,001)$ DIA $\left.^{2} ; C V=13,2 \% ; R^{2}=0,62\right)$. A diferença observada entre os tratamentos alimentares deve-se principalmente ao comportamento da produção de leite das vacas. A produção de leite das vacas mantidas em PN foi linear decrescente sendo de 5,38; 5,01; 4,49; 4,42; 3,72; 3,04; e 1,97 L/dia, respectivamente, aos 14; 42; 70; 98; 126; 154; e 182 dias (Restle et al., 2003). Verifica-se que, a partir dos 98 dias, a queda da produção de leite passou a ser acentuada. Estes resultados mostram que, a partir dos 85 dias, a produção de leite foi insuficiente para que os bezerros mantivessem o mesmo ritmo de ganho de peso. A queda na produção de leite, aliada à queda na qualidade do pasto, resultou na redução do aporte de nutrientes para o bezerro, agravado pelo aumento das exigências de mantença que são propor- cionais ao tamanho metabólico do animal.

Quando os animais foram mantidos em PC, a produção de leite das vacas foi maior, sendo de 4,45; 5,05; 5, 05; 5,10; 5,02; 4,84; e 4,88 L/dia, para os mesmos períodos acima citados, o que, aliado à melhor qualidade do pasto, permitiu que os animais mantivessem elevado ganho de peso por um período mais prolongado. Verifica-se ainda, na Tabela 2 , que a diferença percentual no GMD entre bezerros da PN e PC aumentou acentuadamente a partir dos 98-126 dias, sendo que a diferença de 38\% neste período passou para $190 \%$ no período dos 182 aos 217 dias.

Analisando o efeito do tipo de pastagem, verifica-se que bezerros mantidos em PC ganharam, em média, $263 \mathrm{~g} /$ dia a mais $(\mathrm{P}=0,0001)$ que bezerros mantidos em PN (715 vs 452 g/dia), refletindo significativamente $(\mathrm{P}=0,0001)$ em maior peso ao desmame para os primeiros (180,2 vs 130,5 kg). Moojen et al. (1994 b) verificaram que os bezerros que permaneceram com as vacas em PC apresentaram ganho de peso superior (670 g/dia) em relação aos que permaneceram com as vacas em PN (381 g/dia), o que está de

Tabela 1 - Médias para peso $(\mathrm{kg})$ dos bezerros, de acordo com o tipo de pastagem, grupo genético da vaca e período pós-parto

Table 1 - Means for calves weight $(\mathrm{kg})$, according to pasture, cow genetic group and postpartum period

\begin{tabular}{|c|c|c|c|c|c|c|c|c|c|c|}
\hline \multirow[t]{2}{*}{$\begin{array}{l}\text { Pastagem } \\
\text { Pasture }\end{array}$} & \multirow{2}{*}{$\begin{array}{c}\text { Grupo } \\
\text { Genético } \\
\text { da vaca } \\
\text { Cow } \\
\text { genetic } \\
\text { group }\end{array}$} & \multicolumn{9}{|c|}{$\begin{array}{l}\text { Período pós-parto (dias) } \\
\text { Postpartum period (days) }\end{array}$} \\
\hline & & $\begin{array}{c}\text { Nascer }^{+} \\
\text {Birth }^{+}\end{array}$ & 14 & 42 & 70 & 98 & 126 & 154 & 182 & $\begin{array}{l}\text { Desmame } \\
\text { Weaning }\end{array}$ \\
\hline \multirow{3}{*}{$\begin{array}{l}\text { Cultivada } \\
\text { Cultivated }\end{array}$} & Charolês & 36,3 & 45,1 & 64,9 & 86,9 & 113,6 & 134,7 & 162,8 & 182,0 & 189,2 \\
\hline & $\begin{array}{c}\text { Charolais } \\
\text { Nelore } \\
\text { Nellore }\end{array}$ & 30,2 & 36,7 & 49,4 & 71,8 & 93,5 & 115,7 & 140,2 & 158,9 & 171,2 \\
\hline & $\begin{array}{l}\text { Média* } \\
\text { Mean }\end{array}$ & 33,3 & 40,9 & 57,1 & 79,3 & 103,6 & 125,2 & 151,5 & 170,5 & $180,2 \mathrm{a}$ \\
\hline \multirow[t]{3}{*}{$\begin{array}{l}\text { Nativa } \\
\text { Native }\end{array}$} & $\begin{array}{l}\text { Charolês } \\
\text { Charolais }\end{array}$ & 36,4 & 41,5 & 55,2 & 71,4 & 88,3 & 104,5 & 119,6 & 131,2 & 137,8 \\
\hline & $\begin{array}{l}\text { Nelore } \\
\text { Nellore }\end{array}$ & 30,6 & 34,5 & 47,3 & 63,5 & 80,1 & 96,9 & 113,0 & 121,5 & 123,2 \\
\hline & $\begin{array}{l}\text { Média** } \\
\text { Mean }\end{array}$ & 33,5 & 38,0 & 51,3 & 67,5 & 84,2 & 100,7 & 116,3 & 126,3 & $130,5 b$ \\
\hline
\end{tabular}

* $y=30,085( \pm 0,484)+0,739( \pm 0,004) D I A * ; C V=4,69 \% ; R^{2}=0,99 ; P=0,0001$

** $y=33,858( \pm 0,457)+0,492( \pm 0.004) D I A ; C V=5,64 \% ; R^{2}=0,98 ; P=0,0001$

\# Dia pós-parto (Postpartum day).

+ Retirado efeito de tipo de pastagem (Pasture effect was removed)

$a, b$ Médias seguidas por letras minúsculas diferentes na coluna diferem $(P<0,05)$ pelo teste $F$

$a, b \quad$ Means followed by different small letters in the column differ $(P<.05)$ by $F$ test. 
acordo com os resultados obtidos por Costa et al. (1981), em que bezerros mantidos em PC foram desmamados aos 217 dias com $180 \mathrm{~kg}$, enquanto os mantidos em PN, com $148 \mathrm{~kg}$. No estudo de Senna (1996), o autor avaliou o efeito da PN ou PC sobre o peso à desmama dos bezerros e obteve os valores de 131 e 146 kg, respectivamente.

As correlações entre a produção de leite das vacas e os pesos e ganhos de peso médio diários dos bezerros ao longo do período pós-parto são apresentadas na Tabela 3. Nota-se, de maneira geral, que as correlações tanto para peso como para GMD foram significativas mesmo nos períodos mais avançados da lactação, indicando uma relação marcante entre a oscilação na produção de leite e estas duas variáveis. Comentários discordantes foram feitos por Robison et al. (1978) e Leal \& Freitas (1982) de que a dependência do leite é bastante marcante nos primeiros quatro meses de vida do bezerro, diminuindo a correlação entre produção de leite e ganho de peso diário a partir desta idade, devido à taxa de ganho de peso do bezerro depender mais da ingestão de forragem que da ingestão de leite. Porém, Ribeiro \& Restle (1991) verificaram correlações significativas e altas até o desmame, assim como Drewry et al.
(1959), trabalhando com animais Angus por 203 dias pós-parto, sendo de -0,15; 0,35; e 0,48 para primeiro, terceiro e sexto mês, respectivamente, concordando com os resultados obtidos no presente experimento.

Considerando a produção média diária de leite dos 14 aos 182 dias, a correlação com o peso e com o GMD dos bezerros $\mathrm{CH}, 1 / 2 \mathrm{CH} 1 / 2 \mathrm{NE}$ e $1 / 2 \mathrm{NE} 1 / 2 \mathrm{CH}$ foram superiores àquelas dos bezerros NE. Este resultado indica que bezerros $\mathrm{CH}$ e $\mathrm{F} 1$ foram mais sensíveis às variações da produção de leite de suas mães, porque suas exigências energéticas são maiores devido ao elevado potencial para ganho de peso. Estes comentários são válidos inclusive para explicar a maior correlação nos bezerros filhos de vacas $\mathrm{CH}$ em relação aos filhos de vacas NE, e dos bezerros F1 em relação aos puros. Analisando o tipo de pastagem, a correlação foi maior, tanto para peso quanto para GMD, quando os bezerros foram mantidos em pastagem cultivada.

No presente experimento, a produção média diária de leite das vacas explicou $45 \%$ do peso à desmama e $38 \%$ do ganho de peso dos bezerros. Resultados superiores foram obtidos por Ribeiro \& Restle (1991), em condições sul-brasileiras, onde segundo os autores, a produção de leite explicou 56\% do ganho de peso do nascimento ao desmame, que

Tabela 2 - Médias para ganho de peso médio diário (g) dos bezerros, de acordo com o tipo de pastagem, grupo genético da vaca e período pós-parto

Table 2 - Means for calves average daily weight gain (g), according to pasture, cow genetic group and postpartum period

\begin{tabular}{|c|c|c|c|c|c|c|c|c|c|c|}
\hline \multirow{2}{*}{$\begin{array}{l}\text { Pastagem } \\
\text { Pasture }\end{array}$} & \multirow{2}{*}{$\begin{array}{c}\text { Grupo genético } \\
\text { da vaca } \\
\text { Cow genetic } \\
\text { group }\end{array}$} & \multicolumn{9}{|c|}{$\begin{array}{l}\text { Período pós-parto (dias) } \\
\text { Postpartum period (days) }\end{array}$} \\
\hline & & $0-14$ & $14-42$ & $42-70$ & $70-98$ & $98-126$ & $126-154$ & $154-182$ & $\begin{array}{l}\text { 182-Desm. } \\
\text { Weaning }\end{array}$ & $\begin{array}{l}\text { Média } \\
\text { Mean }\end{array}$ \\
\hline Cultivada & Charolês & 758 & 699 & 777 & 946 & 744 & 996 & 677 & 309 & 738 \\
\hline \multirow[t]{3}{*}{ Cultivated } & Charolais & & & & & & & & & \\
\hline & $\begin{array}{l}\text { Nelore } \\
\text { Nellore }\end{array}$ & 674 & 448 & 793 & 770 & 786 & 867 & 662 & 527 & 691 \\
\hline & $\begin{array}{l}\text { Média } \\
\text { Mean }\end{array}$ & 716 & 573 & 785 & 858 & 765 & 932 & 670 & 418 & $715 a$ \\
\hline Nativa & Charolês & & & & & & & & & \\
\hline \multirow[t]{3}{*}{ Native } & Charolais & 634 & 449 & 535 & 563 & 537 & 496 & 373 & 241 & 479 \\
\hline & $\begin{array}{l}\text { Nelore } \\
\text { Nellore } \\
\text { Média }\end{array}$ & 399 & 430 & 556 & 568 & 573 & 552 & 278 & 046 & 425 \\
\hline & Mean & 517 & 440 & 545 & 566 & 555 & 524 & 325 & 144 & $452 b$ \\
\hline \multicolumn{2}{|c|}{$\begin{array}{l}\text { Média das duas pastagens } \\
\text { Mean of the two pastures }\end{array}$} & 617 & 507 & 665 & 712 & 660 & 728 & 498 & 281 & $*$ \\
\hline
\end{tabular}

* $y=489,89+3,96 \mathrm{DIA}-0,0198 \mathrm{DIA}^{2} ; \mathrm{CV}=9,8 \% ; \mathrm{R}^{2}=0,89 ; \mathrm{P}=0,0001$

a,b Médias seguidas por letras minúsculas diferentes na coluna, diferem $(P<0,05)$ pelo teste $F$.

$a, b$ Means followed by different small letters in the column, differ $(P<.05)$ by $F$ test. 


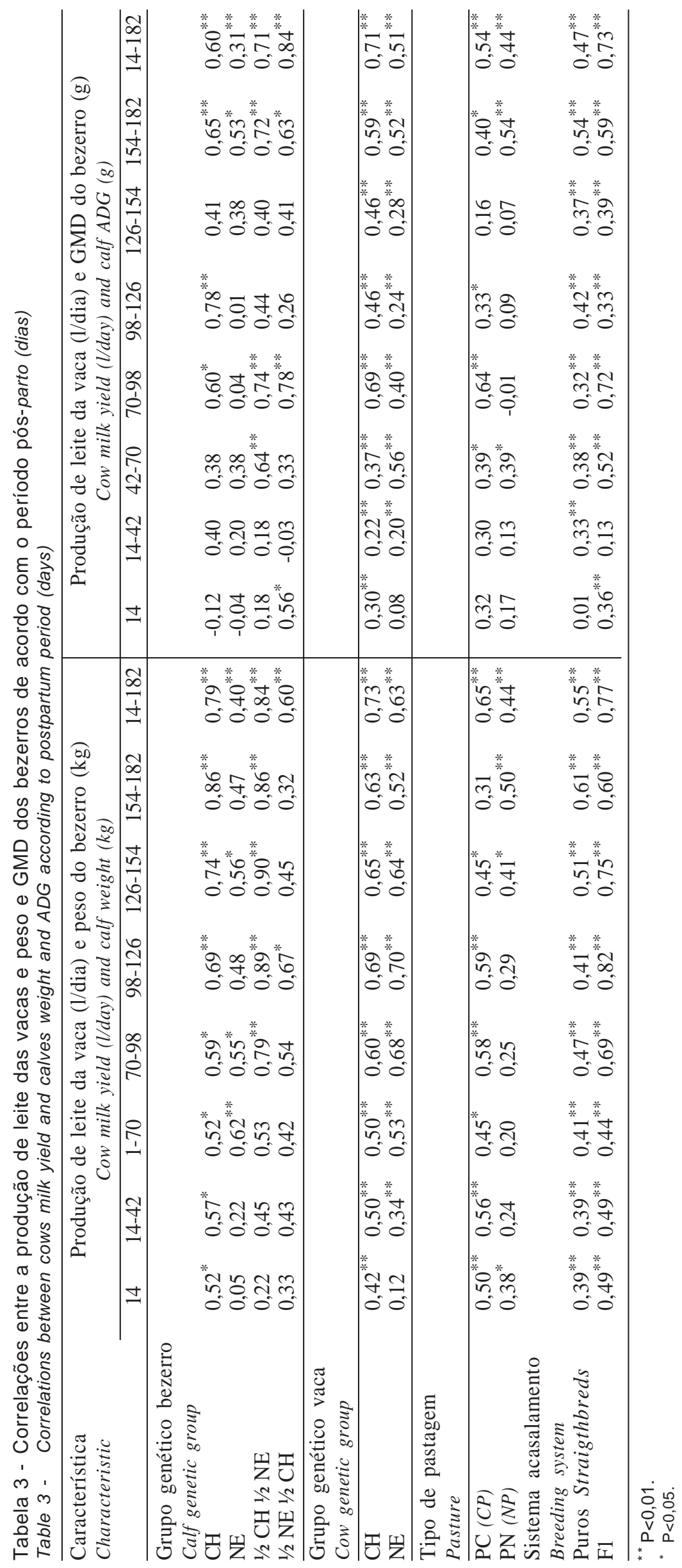


ocorreu aos 235 dias.

Vários estudos demonstraram que a correlação entre a produção de leite da vaca e o peso ao desmame dos bezerros são positivas e altas (Neville Jr., 1962; Rutledge et al., 1971; Totusek et al., 1973; Robison et al., 1978; Alencar et al., 1985), indicando a importância desta característica para o desenvolvimento do bezerro.

As correlações gerais entre o peso e o GMD dos bezerros e as percentagens médias dos 14 aos 182 dias dos componentes do leite foram pouco significativas e próximas de zero. No estudo de Melton et al. (1967) foram verificadas correlações entre -0,01 e 0,03 e no de Ribeiro \& Restle (1991), entre -0,07 e 0,14 (do nascimento ao desmame), confirmando os resultados obtidos no presente estudo. Porém, quando se avaliaram as correlações entre a quantidade total dos componentes do leite $(\mathrm{kg})$ e o GMD e o peso (Tabela 4), todas foram significativas $(\mathrm{P}<0,01)$, concordando com os resultados de Rutledge et al. (1971), que avaliaram por 205 dias animais Hereford, e Totusek et al. (1973), que trabalharam por 210 dias com animais de raças britânicas. De acordo com Melton et al. (1967), a relação do ganho de peso do bezerro com as produções de gordura, sólidos nãogordurosos e sólidos totais foram 0,31; 0,43; e 0,41, enquanto Ribeiro \& Restle (1991), trabalhando com Charolês e Angus, verificaram 0,63; 0,72; e 0,73, respectivamente.

As correlações gerais indicaram que a quantidade de gordura, extrato seco total, extrato seco desengordurado e lactose explicaram, respectivamente, 24 ; 41 ; 35 ; e $40 \%$ do peso e 26 ; 38 ; 35 ; e $37 \%$ do GMD dos bezerros. Nota-se que a quantidade de gordura foi a menos correlacionada, concordando com os resultados reportados por Melton et al. (1967) e Ribeiro \& Restle (1991).

Na Tabela 5, são apresentados os valores médios e heterose para peso ao nascer, ao desmame, e ganho de peso dos bezerros de acordo com o sistema de acasalamento e grupo genético.

Analisando o peso ao nascer, verifica-se que bezerros $\mathrm{CH}$ foram mais pesados (35,0 kg) em relação aos bezerros NE (29,5 kg), assim como bezerros F1 filhos de vacas $\mathrm{CH}(35,8 \mathrm{~kg})$ em relação aos F1 filhos de vacas NE (31,0 kg). Peso ao nascer é uma característica importante, pois está relacionado principalmente com a ocorrência de partos distócitos. De acordo com Hafez (1963) e Winks et al. (1978), o peso ao nascer depende, principalmente, do sexo e grupo genético do bezerro, do ambiente materno, da duração do período de gestação e da época de nascimento.

No estudo realizado por Pereira et al. (2000), que avaliaram o desenvolvimento ponderal de bovinos de corte de diferentes grupos genéticos $\mathrm{CH}$ e $\mathrm{NE}$, os resultados foram semelhantes aos obtidos no presente experimento, em que bezerros $\mathrm{CH}$ apresentaram maior peso $(35,0 \mathrm{~kg})$ que os $\mathrm{NE}(25,0 \mathrm{~kg})$ ao nascer, o que está de acordo com Senna (1996), que verificou $36 \mathrm{~kg}$ para bezerros CH e $29 \mathrm{~kg}$ para os NE. Segundo os autores, o maior tamanho do bezerro $\mathrm{CH}$ se deve ao efeito genético aditivo para esta característica, ao maior tamanho da cavidade abdominal da vaca $\mathrm{CH}$, que permite maior desenvolvimento do feto, e ao maior fluxo sangüíneo no útero das raças européias em relação às zebuínas.

Conforme verificado na Tabela 5, houve diferença significativa no peso ao desmame e no ganho de peso do nascimento ao desmame na comparação entre os grupos genéticos puros. Bezerros $\mathrm{CH}$ ganharam $46 \mathrm{~g}$ a mais por dia (591 vs $545 \mathrm{~g} / \mathrm{dia}$ ) e foram $15,1 \mathrm{~kg}$ mais pesados ao desmame $(156,3 \mathrm{vs}$ $141,23 \mathrm{~kg}$ ) que bezerros NE, assim como bezerros $1 / 2 \mathrm{NE} 1 / 2 \mathrm{CH}$ foram desmamados com superioridade de 8,4 kg (166,3 vs 157,9 kg) em relação aos 1/2 CH $1 / 2 \mathrm{NE}$ embora similares quanto ao ganho de peso médio diário (média de 626 g/dia). Esta superioridade numérica dos bezerros filhos de vacas $\mathrm{CH}$ é explicada pelos efeitos associados do elevado potencial para ganho de peso característico desta raça (Moletta \& Restle, 1992) e pela relação entre o peso à desmama e peso ao nascer do bezerro, pois, segundo comentários de Neville Jr. (1962) e Rutledge et al. (1971), bezerros que nascem mais pesados mantêm a diferença de peso até o desmame ou apresentam ganhos de peso significativamente maiores até o desmame.

Ainda na Tabela 5, verifica-se que os valores de heterose não foram significativos para peso ao nascer dos bezerros. Conclusão semelhante foi relatada por Pereira et al. (2000) avaliando animais CH, NE e suas cruzas F1, com valor de heterose de 3,3\%. Porém, em estudo realizado por Koch (1972), foram verificados valores de heterose variando de 5,0 a 10,8 \%, e Long (1980), ocorrência de até $20 \%$ de heterose individual dos bezerros F1.

Houve heterose significativa $(\mathrm{P}=0,0001)$ para peso ao desmame de $11,8 \%$ e ganho de peso do nascimento ao desmame de $10,2 \%(P=0,0001)$, o que 


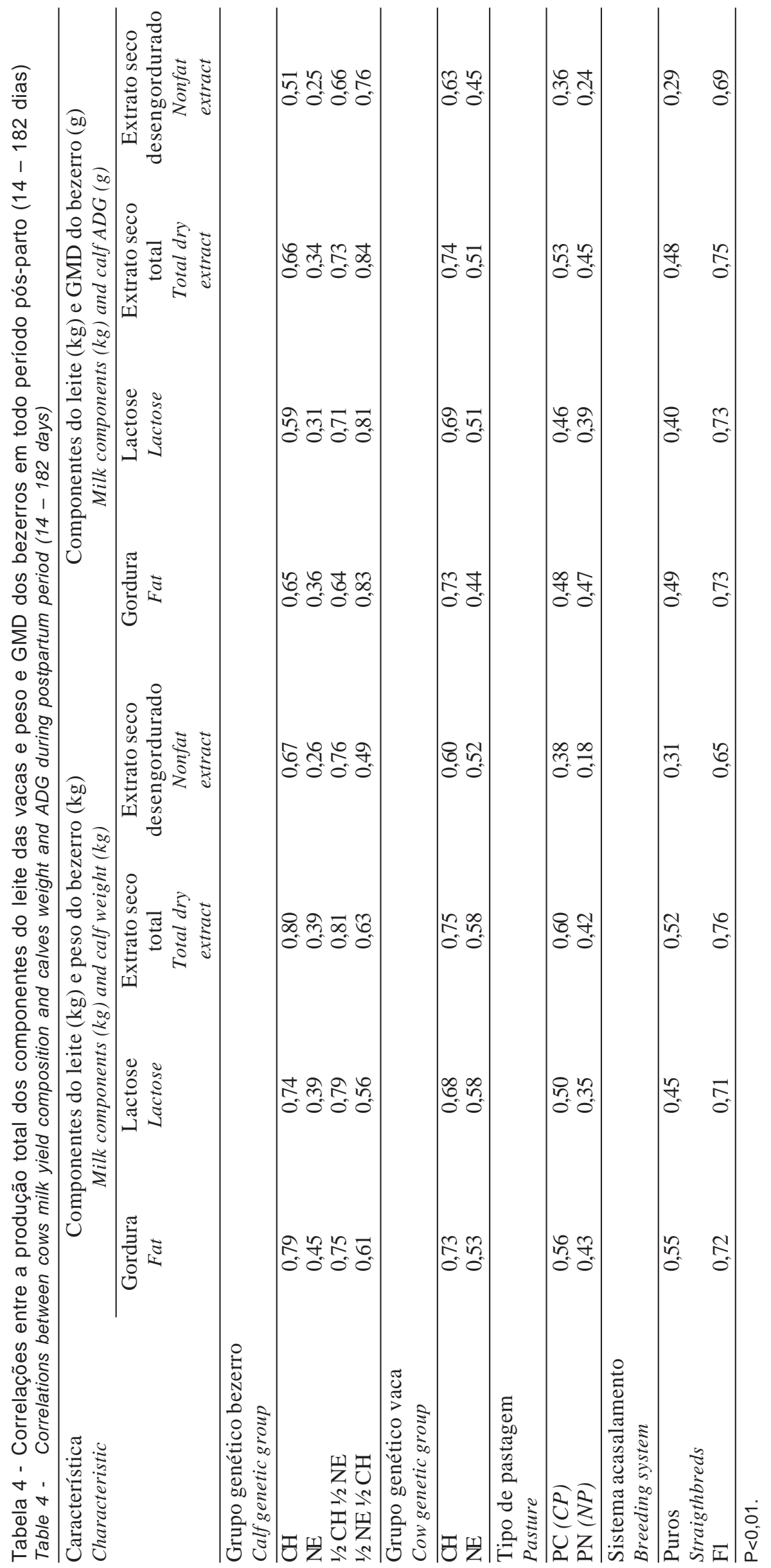


está de acordo com Ribeiro \& Restle (1991) e Senna (1996), que verificaram maiores pesos e ganhos de peso ao desmame para bezerros mestiços em relação aos puros. Valor semelhante de heterose $(12,6 \%)$ para peso dos bezerros aos 8 meses foram relatados por Pereira et al. (2000), embora os autores tenham verificado valor superior quanto ao ganho de peso dos 3-8 meses de idade (38,8\%), avaliando bezerros de mesma composição racial do presente estudo. Estudando o cruzamento das raças $\mathrm{CH}$ e NE, Trematore et al. (1998) observaram heterose de 5,1 e 8,7\% para peso ao nascer e peso ao desmame, respectivamente, concordando com os estudos de Olson et al. (1993) e Perotto et al. (1998), que verificaram heteroses positivas nos pesos para idade até 8 meses, nos cruza- mentos de animais $\mathrm{CH}$ x Brahman e CH x Caracu, respectivamente. Peacock et al. (1981) verificaram, para peso ao desmame, heterose de $21,2 \%$ para as cruzas Aberdeen Angus x Brahman; 1,4\% para as cruzas Aberdeen Angus x $\mathrm{CH}$; e 16,5\% para as cruzas Brahman x $\mathrm{CH}$.

Alguns estudos relatam que as diferenças no desempenho entre os grupos genéticos podem ocorrer pelas diferentes exigências energéticas para mantença e ganho de peso, sendo que genótipos maiores e/ou selecionados para rápido ganho de peso apresentam maiores exigências e aumentam o consumo de alimentos para atenderem a estes requerimentos (Koger, 1973; NRC, 1996). Como no presente estudo o grupo genético do bezerro não influenciou a produção de

Tabela 5 - Médias e heterose para peso ao nascer, ao desmame e ganho de peso médio diário (GMD) dos bezerros, de acordo com o sistema de acasalamento e grupo genético do bezerro

Table 5 - Means and heterosis for live weight at birth, at weaning and average daily weight gain (ADG) of calves, according to the breeding system and calf genetic group

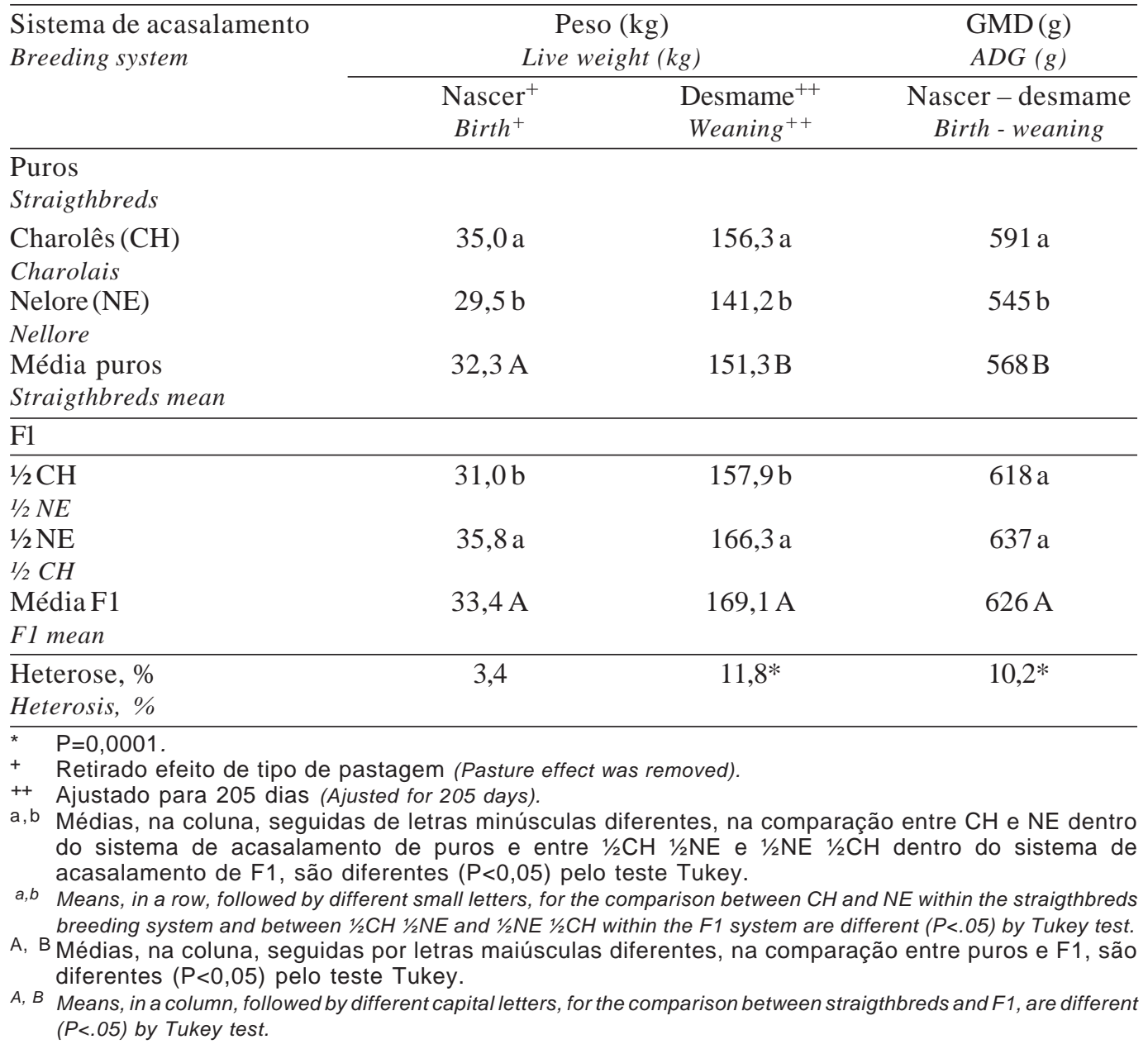


leite das vacas, isto sugere que a contribuição energética do leite na dieta dos bezerros foi semelhante entre os grupos genéticos, demonstrando que as conclusões dos autores acima citados são válidas para explicar as diferenças de desempenho dos bezerros verificadas neste estudo, ou seja, provavelmente bezerros $\mathrm{CH}$ e $\mathrm{F} 1$ tiveram que iniciar o pastejo mais cedo e com maior intensidade para atenderem suas maiores necessidades energéticas em relação aos bezerros NE. Vários trabalhos demonstraram efeito heterótico positivo no consumo de matéria seca (kg), na comparação entre animais puros (CH e NE) e suas cruzas recíprocas F1, como o de Restle \& Alves Filho (1992), na fase de recria em confinamento (6,4\%), e Casaccia et al. (1993) e Restle et al. (1995), na fase de terminação em confinamento (13,6 e $15,4 \%$, respectivamente). No estudo de Melton et al. (1967), foi verificado que bezerros $\mathrm{CH}$ foram menos eficientes na transformação de leite em ganho de peso devido a seu elevado requerimento de mantença comparado com bezerros Hereford e Angus.

O peso ao nascer, ao desmame e o ganho de peso não foram influenciados pelo sexo do bezerro, apesar de os machos terem sido numericamente $5,3 \%$ mais pesados (164,5 vs 155,8 kg) ao desmame e ganharem $4,8 \%$ mais peso/dia (601 vs 572 g/dia) em relação às fêmeas. Alguns trabalhos relatam maiores ganhos de peso e pesos ao desmame para os machos em relação às fêmeas, como os de Melton et al. (1967), Bond \& Wiltbank (1970), Rutledge et al. (1971), Cundiff et al. (1974) e Reynolds et al. (1978). Em seu estudo, Ribeiro \& Restle (1991) verificaram que os machos foram sempre mais pesados que as fêmeas e tiveram maior ganho de peso médio diário (0,595 vs 0,472 g/ dia, respectivamente). Porém, similaridade entre os sexos para peso à desmama foi relatada por Bellows \& Short (1978) e Rocha \& Ribeiro (1987).

Não foi verificada interação significativa entre classe de idade da vaca e os demais efeitos para peso ao nascer, ao desmame e ganho de peso. Contudo, nota-se que houve influência da classe de idade da vaca $(\mathrm{P}=0,0549)$ no peso ao nascer dos bezerros. Vacas jovens pariram bezerros mais leves $(30,2 \mathrm{~kg})$ que vacas adultas $(34,1 \mathrm{~kg}$ ) e velhas (34,2 kg). Porém, quando este peso é ajustado por unidade de peso metabólico das vacas, estas diferenças passaram a ser menores $(\mathrm{P}=0,0710)$, com valores de 380,0; 382,2; 393,0 g, respectivamente, para vacas jovens, adultas e velhas. Isto demonstra que o peso dos bezerros apresenta grande relação com o peso das vacas.

A classe de idade não afetou o ganho de peso dos bezerros (média de 586,3 g/dia). Porém, vacas adultas desmamaram bezerros mais pesados $(174,2 \mathrm{~kg})$ em relação às vacas jovens $(145,3 \mathrm{~kg})$, com o peso dos bezerros de vacas velhas atingindo valor intermediário (170,0 kg). Resultados de vários estudos citam que os maiores pesos à desmama dos bezerros coincidem com as maiores produções de leite quando as vacas atingem a maturidade (Rutledge et al., 1971; Neville Jr. et al., 1974; Rovira, 1974). Embora a produção média diária de leite das vacas não tenha diferido entre as classes de idade, no presente estudo, o que poderia explicar as diferenças no peso dos bezerros ao desmame seria a produção total de leite, que diferiu significativamente $(\mathrm{P}=0,0001)$, sendo superior nas vacas adultas $(991,5 \mathrm{~L})$ em relação às jovens $(877,1 \mathrm{~L})$ e velhas (779,3 L). Outros fatores que provavelmente contribuíram para as diferenças no peso à desmama seriam as diferenças observadas, significativamente, no peso ao nascer e, numericamente, no GMD.

\section{Conclusões}

Houve benefício da melhoria das condições alimentares no desenvolvimento dos bezerros, pois o uso de pastagem cultivada resultou em animais mais pesados à desmama, reflexo do elevado ganho de peso médio diário, quando comparados com os mantidos em pastagem nativa.

Bezerros filhos de vacas Charolês foram mais pesados ao nascer e à desmama que os filhos de vacas Nelore.

Bezerros F1 apresentaram desempenho superior aos puros, indicando que o cruzamento constitui uma importante ferramenta no incremento da eficiência produtiva de um sistema de ciclo completo.

As correlações demonstraram que bezerros com maior potencial para ganho de peso foram mais sensíveis às variações na produção de leite e à produção total dos componentes do leite das vacas. 


\section{Literatura Citada}

ALENCAR, M.M.; FILHO, A.A.J.; PARANHOS, N.E. Produção de leite das vacas e desenvolvimento dos bezerros em um rebanho Canchim. In: REUNIÃO ANUAL DA SOCIEDADE BRASILEIRA DE ZOOTECNIA, 22., 1985, Camboriú. Anais... Viçosa, MG: Sociedade Brasileira de Zootecnia, 1985. p.223.

ASSOCIATION OF OFFICIAL ANALYTICAL CHEMISTS AOAC. Official methods of analysis. 14.ed. Washington, D.C.: 1984. 1141p.

BARCELLOS, J.O.J.; LOBATO, J.F.P. Efeitos da época de nascimento no desenvolvimento de bezerros Hereford e suas cruzas. I. Peso ao nascer e ganho médio diário pré-desmama. Revista da Sociedade Brasileira de Zootecnia, v.21, n.1, p.137-149, 1992.

BECKER, A.S.; HILLERBRAND, G.L.; POLLI, V.A. et al. Desempenho de fêmeas “Threecross” e Hereford. III. Características com o terneiro. Pesquisa Agropecuária Gaúcha, v.1, n.1, p.103-109, 1995.

BELLOWS, R.A.; SHORT, R.E. Effects of pre-calving feed level on birth weight, calving difficult and subsequent fertility. Journal of Animal Science, v.46, n.6, p.1522-1528, 1978.

BOND, J.; WILTBANK, J.N. Effect of energy and protein on estrus, conception rate, growth and milk production of beef females. Journal of Animal Science, v.30, n.3, p.438-444, 1970.

CASACCIA, J.L.; PIRES, C.C.; RESTLE, J. Confinamento de bovinos inteiros ou castrados de diferentes grupos genéticos. In: REUNIÃO ANUAL DA SOCIEDADE BRASILEIRA DE ZOOTECNIA, 30., 1993, Rio de Janeiro. Anais... Rio de Janeiro: Sociedade Brasileira de Zootecnia, 1993. p.468.

COSTA, A.M.; RESTLE, J.; MÜLLER, L. Influência da pastagem cultivada no desempenho reprodutivo de vacas com cria ao pé. Revista do Centro de Ciências Rurais, v.11, n.4, p.187-200, 1981.

CROCKETT, J.R.; KOGER, M.; FRANKE, D.E. Rotational crossbreeding of beef cattle: preweaning traits by generation. Journal of Animal Science, v.46, n.5, p.1170-1177, 1978.

CUNDIFF, L.V.; GREGORY, K.E.; SCHWULST, F.J. et al. Effects of heterosis on maternal performance and milk production in Hereford, Angus and Shorthorn cattle. Journal of Animal Science, v.38, p.728-745, 1974.

DREWRY, K.J.; BROWN, C.J.; HONEA, R.S. Relationships among factors associated with mothering ability in beef cattle. Journal of Animal Science, v.18, p.938-946, 1959.

HAFEZ, E.S.E. Symposium on growth: physio-genetics of prenatal and postnatal growth. Journal of Animal Science, v.22, p.779-791, 1963.

KOCH, R.M. The role of maternal effects in animal breeding: VI. Maternal effects in beef cattle. Journal of Animal Science, v.35, p.1316-1326, 1972.

KOGER, M. Summary. In: KOGER, M.; CUNHA, T.J.; WARNICH, A.C. (Eds.) Crossbreeding beef cattle. Series 2. Gainesville: University of Florida Press, 1973. p.434-453.

LEAL, T.C.; FREITAS, J.E. Correlação entre produção de leite e ganho de peso de bezerros da raça Charolesa. Anuário Técnico do IPZFO, v.9, p.91-101, 1982.

LONG, C.R. Crossbreeding for beef production: experimental results. Journal of Animal Science, v.51, p.1197-1220, 1980.

MELTON, A.A.; RIGGS, J.K.; NELSON, L.A. et al. Milk production, composition and calf gains of Angus, Charolais and Hereford cows. Journal of Animal Science, v.26, p.804-809, 1967.

R. Bras. Zootec., v.33, n.3, p.691-703, 2004
MOLLETA, J.L.; RESTLE, J. Desempenho em confinamento de novilhos de diferentes grupos genéticos. Ciência Rural, v.22, n.2, p.227-233, 1992.

MOOJEN, J.L.; FELTEN, H.G.; RESTLE, J. et al. Efeito da época da desmama e da pastagem no desempenho de vacas e terneiros de corte. 1. Desempenho de vacas. Ciência Rural, v.24, n.2, p.393-397, 1994a.

MOOJEN, J.L.; RESTLE, J.; MOOJEN, E.L. et al. Efeito da época da desmama e da pastagem no desempenho de vacas e terneiros de corte. 1. Desempenho de terneiros. Ciência Rural, v.24, n.2, p.399-403, 1994b.

NATIONAL RESEARCH COUNCIL - NRC. Nutrient requeriments of beef cattle. 6.ed. Washington, D.C.: National Academy Press, 1996. 242p.

NEVILLE JR., W.E. The influence of dam's milk production and other factors on 120- and 240-day weight of Hereford calves. Journal of Animal Science, v.21, p.315, 1962.

NEVILLE JR., W.E.; WARREN, E.P.; GRIFFEY, W.A. Estimates of age effects on milk production in Hereford cows. Journal of Animal Science, v.38, v.1, p.1-5, 1974.

OLSON, T.A.; PEACOCK, F.M.; KOGER, M. Reproductive and maternal performance of rotational, three-breed, and Inter se crossbred cows in Florida. Journal of Animal Science, v.71, p. 2322-2329, 1993.

PEACOCK, F.M.; KOGER, M.; OLSON, T.A. et al. Additive genetic and heterosis effects in crosses among cattle breeds of british, european and zebu origin. Journal of Animal Science, v.52, n.5, p.1007-1012, 1981.

PEREIRA, L.P.; RESTLE, J.; BRONDANI, I.L. et al. Desenvolvimento ponderal de bovinos de corte de diferentes grupos genéticos de Charolês x Nelore inteiros ou castrados aos oito meses. Ciência Rural, v.30, n.6, p.1033-1039, 2000.

PEROTTO, D.; CUBAS, A.C.; MOLETTA, J.L. et al. Pesos ao nascimento e à desmama e ganho de peso do nascimento à desmama de bovinos Charolês, Caracu e cruzamentos recíprocos. Revista Brasileira de Zootecnia, v.27, n.4, p.730737, 1998

QUADROS, S.A.F.Q.; LOBATO, J.F.P. Efeitos da lotação animal na produção de leite de vacas de corte primíparas e no desenvolvimento de seus bezerros. Revista Brasileira de Zootecnia, v.26, n.1, p.27-33, 1997.

RESTLE, J.; ALVES FILHO, D.C. Confinamento de terneiros inteiros ou castrados de diferentes grupos genéticos. In: REUNIÃO ANUAL DA SOCIEDADE BRASILEIRA DE ZOOTECNIA, 29., 1992, Lavras. Anais... Lavras: Sociedade Brasileira de Zootecnia, 1992. p.186.

RESTLE, J.; BRONDANI, I.L.; BERNARDES, R.A.C. O novilho superprecoce. In: RESTLE, J. (Ed.) Confinamento, pastagens e suplementação para produção de bovinos de corte. Santa Maria: Universidade Federal de Santa Maria, 1999b. p.191-214.

RESTLE, J.; FELTEN, H.G.; VAZ, F.N. Efeito de raça e heterose para desempenho em confinamento de novilhos de corte. In: REUNIÓN LATINOAMERICANA DE PRODUCCIÓN ANIMAL, 14., 1995, Mar del Plata. Memórias... Balcarce: ALPA, 1995, v.3-4, p.852-854.

RESTLE, J.; PACHECO, P.S.; MOLETTA, J.L. et al. Grupo genético e nível nutricional pós-parto na produção e composição do leite de vacas de corte. Revista Brasileira de Zootecnia, v.32, n.3, p.585-597, 2003.

RESTLE, J.; POLLI, V.A.; SENNA, D.B. Efeito de grupo genético e heterose sobre a idade e peso à puberdade e sobre o desempenho reprodutivo de novilhas de corte. Pesquisa 
Agropecuária Brasileira, v.34, n.4, p.701-707, 1999a.

REYNOLDS, W.L.; DeROUEN, T.M.; BELLOWS, R.A. Relationships of milk yield of dam to early growth rate os straightbred and crossbred calfs. Journal of Animal Science, v.47, n.3, p.584-594, 1978.

RIBEIRO, E.L.A.; RESTLE, J. Desempenho de terneiros Charolês e Aberdeen Angus puros e seus mestiços com Nelore. Pesquisa Agropecuária Brasileira, v.26, n.8, p.1145-1151, 1991.

RIBEIRO, E.L.A.; RESTLE, J.; PIRES, C.C. Produção e composição do leite de vacas Charolês e Aberdeen Angus amamentando bezerros puros ou mestiços. Pesquisa Agropecuária Brasileira, v.26, n.8, p.1267-1273, 1991.

ROBISON, O.W.; YUSUFF, M.K.M.; DILLARD, E.U. Milk production in Hereford cows I. Means and correlations. Journal of Animal Science, v.47, n.1, p.131-135, 1978.

ROCHA, M.A.; RIBEIRO, E.L.A. Influência do cruzamento sobre o peso ao desmame em bovinos de corte. In: SEMINÁRIO DE PESQUISA D AUNIVERSIDADE ESTADUAL DE LONDRINA, 4., 1987, Londrina. Anais... Londrina: FUEL, 1987, p.101.

ROVIRA, J. Reproduccion y manejo de los rodeos de cria. Montevidéo: Hemisfério Sur, 1974. 293p.

RUTLEDGE, J.J.; ROBISON, O.W.; AHLSCHWEDE, W.T. et al. Milk yield and its influence on 217-day weight of beef calfs. Journal of Animal Science, v.33, p.563-567, 1971.

SALOMONI, E.; LEAL, J.J.B.; DEL LUCA, L.O.A. et al. Parição de outono: efeito da carga animal no comportamento reprodutivo de vacas Ibagé e no desenvolvimento de suas crias. Bagé: Embrapa, 1982. 23p. (Boletim de Pesquisa, 2)

STATISTICAL ANALYSES SYSTEMS - SAS. User's guide. Version 6, Cary: v.2, 1997.
SENNA, D.B. Desempenho reprodutivo e produção de leite de vacas de quatro grupos genéticos, desterneiradas precocemente, submetidas a diferentes períodos de pastagem cultivada. Santa Maria: Universidade Federal de Santa Maria, 1996, 85p. Dissertação (Mestrado em Zootecnia) - Universidade Federal de Santa Maria, 1996.

TILLEY, J.M.A.; TERRY, R.A. A two-stage technique for the in vitro digestion of forage crop. Journal of British Grassland Society, v.18, n.2, p,104-111, 1963.

TOTUSEK, R.; ARNETT, D.W.; HOLLAND, G.L. et al. Relation of estimation method, sampling interval and milk composition to milk yield of beef cows and calf gain. Journal of Animal Science, v.37, n.1, p.153-158, 1973.

TREMATORE, R.L.; ALENCAR, M.M.; BARBOSA, P.F. et al. Estimativas de efeitos aditivos e heteróticos para características de crescimento pré-desmama em bovinos Charolês - Nelore. Revista Brasileira de Zootecnia, v.27, n.1, p.87-94, 1998.

WILM, H.G.; COSTELLO, D.F.; KLIPPLE, G.E. Estimating forage yield by the double-sampling methods. Journal of American Society of Agronomy, v.36, p.194-203, 1944.

WINKS, L.; O’ROURKE, P.K.; VENEMORE, P.C. et al. Factors affecting birth weight and performance to weaning of beef calves dry tropics on North Queensland. Australian Journal of Experimental Agriculture and Animal Husbandry, v.18, p.494-499, 1978. 